\title{
Advance Driver Assistance System for Indian Road and Traffic Scenario
}

\footnotetext{
${ }^{1}$ Mr. Abhijit S. Titarmare, ${ }^{2}$ Dr. Milind M. Khanapurkar, ${ }^{3}$ Miss. Indrayani B. Dhotre

${ }^{1}$ Research Scholar, Electronics Engineering Department, G. H. Raisoni College of Engineering, Nagpur, India

${ }^{2}$ Head, Electronics and Telecommunication Engineering, G. H. Raisoni College of Engineering, Nagpur, India

${ }^{3}$ Research Scholar, Electronics and Telecommunication Engineering, Raisoni College of Engineering, Nagpur Email:abhijit.titarmare@raisoni.net ${ }^{1}$,milind.khanapurkar@ raisoni.net ${ }^{2}$, dhotreindrayani@gmail.com ${ }^{3}$
}

\section{Received: 09 ${ }^{\text {th }}$ July 2018, Accepted: $14^{\text {th }}$ August 2018, Published: $31^{\text {st }}$ August 2018}

\begin{abstract}
There are many differences between Indian road and traffic structure compare to foreign road and traffic scenario. Many Driver assistance systems failed in such heavy traffic and congested road structure. The proposed Advanced Driver Assistance System is modified system especially for Indian roads. Road detection is difficult because of poor lane marking and compact roads plans. Because of insufficient space the traffic congestion is more which makes vehicle detection difficult. The proposed system presents the solution for these problems. The road boundaries and edges of drivable lane is detected using Hough Transformation. While on road obstacles are detected from comparing the present and previous frames of video. The safety alerts will be given if other vehicles are too close to car or in Region of Interest (RoI) area. The comparison of road scenario around world is also presented in paper.
\end{abstract}

Keywords: ACC, PCS, LKA, Region of Interest (RoI), U-Y Selector

\section{Introduction}

India is the fastest growing nation. As second populated of nation in the world and due to insufficient transportation space the Indian roads are always loaded with heavy and congested traffic. There is death occur of one person in every four minutes in India due to road accident. Near about 1214 vehicle collisions occurs every day in India [1]. The poor judgment of immature driver increases the higher chances of accidents and crashes. The proposed Advanced Driver Assistance System is helping mechanism for driver. The main purpose of ADAS system is the driver's and vehicle safety. The system follows three main strategies which LKA (Lane Keep Assist), ACC (Adaptive Cruise Control), and PCS (Pre-Crash System). The LKA (lane keep assist) system keep the host vehicle driving inside the roadway, keep eye on road marking and signs on side way and the ACC (adaptive cruise control) is used to calculate distance between front or side cars and alerting driver if he goes too close, and PCS (pre-crash system) will keep alerting host car driver about distance and if driver ignores the alerts the PCS will pull off the emergency breaks automatically. Using these three features the system will alert the driver. The alert will be display on the screen on dashboard. The intensity of traffic is much higher in India compare to aboard. Figure 1 shows the difference between Indian Traffic scenario and USA traffic scenario. The size and shapes of vehicles are also plays important role when it comes to detect on road vehicles. Numbers of two wheelers, three wheelers as well as four wheelers are more in India where in aboard the big transportations are used.

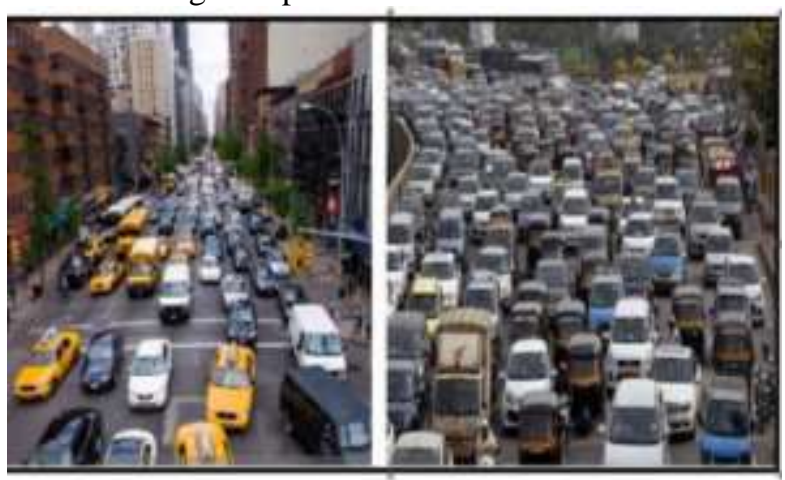

USA Traffic Scenario Delhi, India Traffic

Fig.1: Traffic Comparison between India and USA

The System works in two parts i.e. Car Detection and Road Detection. For detecting the drivable lane, the lane edges and boundaries are calculated using edge detection method. LKA will follow these calculated edges and boundaries and if the driver shifts out of the lane the driver will receive the alert from system that he is going out of drivable road. The edge detection methods like Sobel, Prewitt, Roberts or Canny can be used. Sobel, Prewitt, Roberts detects the edges by approximating the gradients magnitude of a video where as in Canny edges are calculated by finding local maxima of a gradient of input video. By adjusting the threshold value and standard deviation of Gaussian filter the clear edges can be detected. For tracking and detection of on road vehicles the pixel variation between present frame and previous frame is calculated by difference block in MATLAB Simulink. Background estimator is used to detect 
pixels movement in frames. The input video is converted into gray scale to minimize the error ratio. Threshold is used to transfer an intensity image to a binary image. For car tracking, original video and background estimator output is added.

\section{Comparison of Different Adas}

The chaotic traffic makes edges detection and the target recognition difficult and the weather conditions, size and color of the car, road material, also minimize the accuracy. The GPS (Global Positioning System) is popular technology used for tracking and communication purpose in some ADAS. But inaccurate or outdated map data of GPS can cause limitations on temporal and spatial resolution. The high quality and resolution cameras and sensors are placed on the cars for detecting and tracking. The cost map is used to calculate the geological properties of the roads. Graphic Processor Unit (GPU) is use to generate cost map [6].The reliable transportation can be takes place using V2V (vehicle to vehicle) or V2I (Vehicle to Infrastructure) communication system. In vehicleto-vehicle $(\mathrm{V} 2 \mathrm{~V})$ the communication takes place via custom messages and exchange information in a Cooperative system. To avoid an accident or busy routes the alerts about the traffic ahead send to the driver, using these messages and information the driver can takes alternate routes [3]. Vehicle to Infrastructure (V2I) uses an optimization algorithm for personalized pace for approaching and passing signalized intersections. The characteristics and preferences of driver are absorbed by system. While driving system uses this information in approximation of driving pace to boost safety, fuel economy, minimize waiting time, and preferences of driver are addressed [10]. The behavior and preferences of Driver is a major element in road and vehicle safety. By mounting two cameras consists of infrared emitters on the front panel of car which to illustrate user's facial reaction. The camera keeps extracting information such as disturbance in eye blinking, frequency of opening of eyelid, size of pupil position etc. It will only alert driver only if he falls asleep or distracted [7]. The alert to driver can be given by sending signals like short vibrations in steering wheel.

The tracking of cars and edges of road is more difficult in dark. The illuminations like vehicle's lights, street side lamps, and street side bright and shiny sign boards leads to error in the detection and tracking of the cars and road boundaries and edges. At night, level of difficulty increases as finding difference between the moving and non-vehicle illuminations is hard. Effectively sensing and tracking of vehicle and road at night is done by the vision-based intelligent driver assistance system [8]. There is no fully developed ADAS technology in India. The climate and environmental disparities like heat, rain, road condition, traffic management etc. within country makes the implementation difficult. The accurate and reliable ADAS techniques are developed notwithstanding numbers of research, protocols and algorithm. The rural side transportations are congested and chaotic. The unavailability of full road view, it is very difficult to detect distance region and the mid-range region. The vehicles could be on any position. If diver is too close to or surrounded by numbers of other vehicle, then due to less space makes detection and tracking protocols unsuccessful [9].

\section{System Overview}

The overall system is consists of three parts as shown in fig.2:

1. Video Processing Blocks

2. Vehicle and Road Tracking

3. Alerts

In Video Processing Blocks the input video is first limited to pre-defined size using Resize block which uses defined parameters to allocate the parameters user want to resize. The drivable lane and the front cars and other vehicles are main focus of system. To detect the vehicles and lane the Region of Interest (ROI) is generated. The distance of ROI can be set according to user perspective. If any obstacles come in between the ROI, the system will alert the driver to slow down the vehicle. Using the selected region of road the time to calculated drivable roads boundaries and edges is increases as unwanted part is out of focus. U-Y Selector block reorder or select the specified parameters of multidimensional input video and set region of interest. After this to eliminate the illusions of illuminations the colored video is converted into gray scale using Color Conversion Block. The color information between the spaces in colors is converted.

The detection of vehicles and other moving objects are tracked using Difference block. The difference in output of current input value and the previous input value is calculated in this block. The difference in pixels in current and previous frame will locate the position on moving objects. To avoid false detection the unwanted background is masked using Background Estimator block. Background Estimator consists of Temporal Median Estimator and Motion Based Background Estimator. Temporal Median estimator calculates the median values of video data with respect to time. For boost accuracy with new video frames the median value is estimated after 2 frames and Temporal median will compute median value of series of frames after 30 numbers of frames. 
After this computation the motion based estimator will use the first few frames of input video to measure the background image. The subtraction of background of each video frame will create forefront images \& only redraw background section which is in moving position. After all estimation the car is tracked and if it come in ROI the Pre-crash system alert will be given to driver.

In Lane tracking the video is also converted into $\mathrm{YCbCr}$ color space as sensitivity to luminance and chrominance is less in human and this conversion makes easy to get rid the calculation of redundant color information. The using Edge detection block the boundaries of road as well as drivable lane are calculated. 2D FIR Filter is use for stability and linearity of phase. The auto threshold will converts gray scale image to a binary image. The Hough Transform is use to detect these lines. The block generates a constraint space matrix using equation:

$$
\mathrm{Rho}=\mathrm{x} * \cos \theta+\mathrm{y}^{*} \sin \theta
$$

The Line coordinator will track these detected lines using Kalman filter. Hough line block will track all detected edges and then convert lines from polar to Cartesian space. The left and right of lane and boundary of road are calculated using all information.

\section{Methodology}

The Advanced Driver Assistance System works in two parts i.e. Car Detection and Road Detection as shown below.

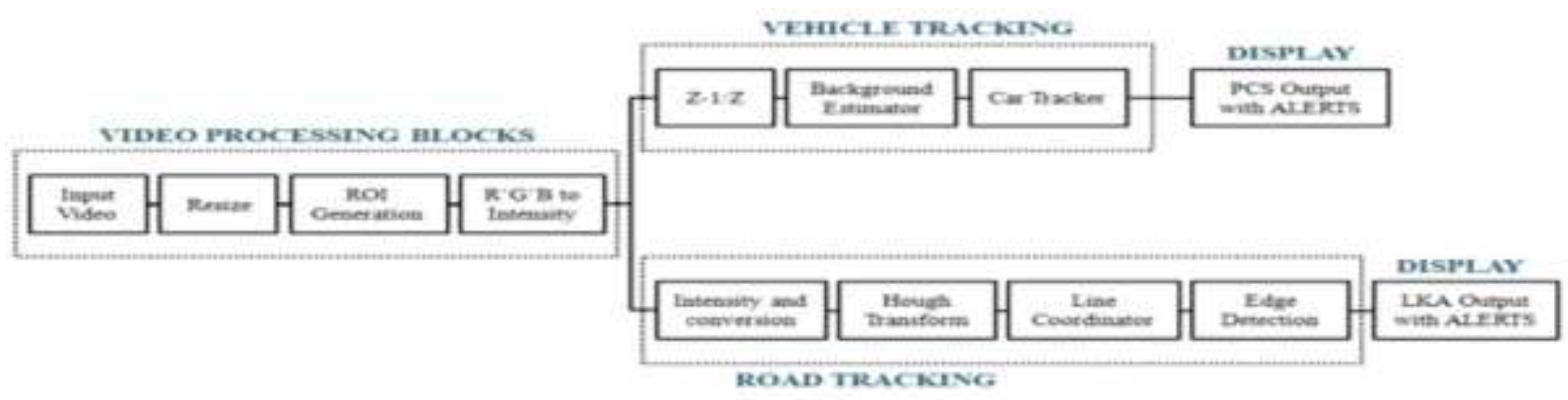

Fig.2: Overview of ADAS

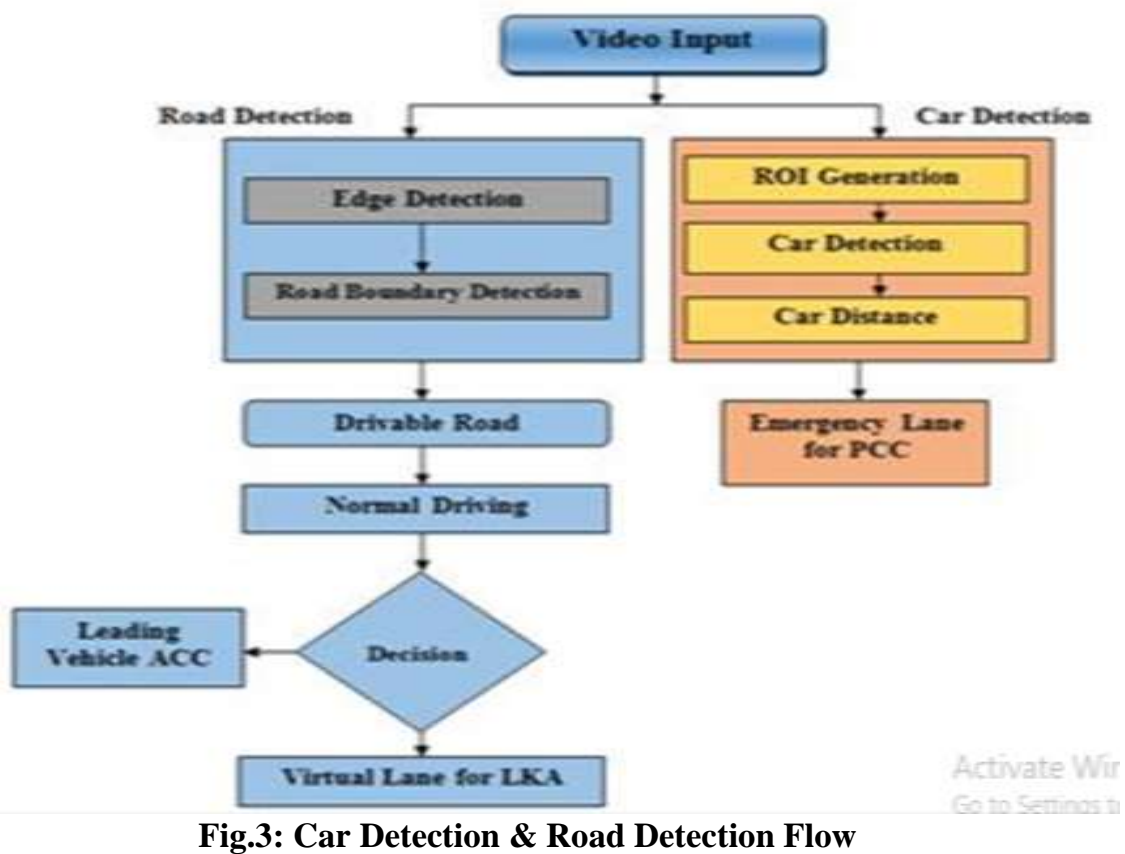

The road detection consists of calculation and tracking the edges and boundaries of road. Then identify the drivable road and normal road. If the car shift out of drivable road or comes near edges of side road then decision will be taken which will alert the driver suggesting the safe position on road. In Car detection RoI is generated to avoid the crash. If any object come across the defined region then system will alert driver and will also suggest the emergency lane for pre-crash control. 


\section{Car Detection}

The car detection is most important function of system because accidents are frequently happened due to human misjudgments. Poor perception while driving leads to crashes or major accidents. For detecting the on-road vehicle the system calculate the position of pixels differences in two video frames. The Difference (MASK) Block is use to calculated the contrast in pixels position. Using formulae Z-1/Z, the block takes the values of current video frame and subtracts it from previous video frame. For getting more accurate result the video is converted into R'G'B' to Gray scale, in this white pixels portion of video frame will show the active pixels. The unwanted background distractions create the errors. To avoid this Background Estimator is used. It consists of Temporal Median Estimator which calculates median values of video data of 2 frames after which the median value will be valid. For gaining reliability Temporal median compute the series of median values after 30 video frames. The Motion Based Background Estimator will take first two-three frames of video and will subtracts the background from each video frame which will construct the foreground picture i.e. moving parts revealed by re-illustrating the moving portion of framework. The pixels in the closed loop will be considered as the obstruction. The Blob Analysis will compute the stats of connected pixels in grey scale binary image. The auto threshold is convert the intensity image to binary image. Connected region having minimum blob area of 500 pixels and maximum blob area of 2500 pixels will considered as closed loop.

Detection part is not only important aspect of the project. The alerts are important to make sure safety. These alerts are decided using Region of Interest (RoI). Selector Block is use to decide the RoI area. If the vehicles, pedestrian or any other obstacles come across the RoI the system will send alerts depending upon the distances of other vehicle from driving vehicle. These alerts are decided by specific algorithm. The RoI is set to be 250:400 pixels, as pixels below 250 is part of vehicle bonnet which is unwanted part.

For Vehicle tracking the video is divided into four different parts as shown in figure 4 . The left and right part of videos is focus on the boundaries and edges of the roads and pedestrians, while middle part and upper middle part focuses only on road. Main advantage of tracking in four parts is the detection will be more accurate as the intensity of traffic in specific part will be track. The white parts of image show the higher intensity of pixels movement. The alerts are allocated depending upon the intensity of movement. For example the system will not give any alert if white part of video is $1<2000$ that means the road is free for driving.

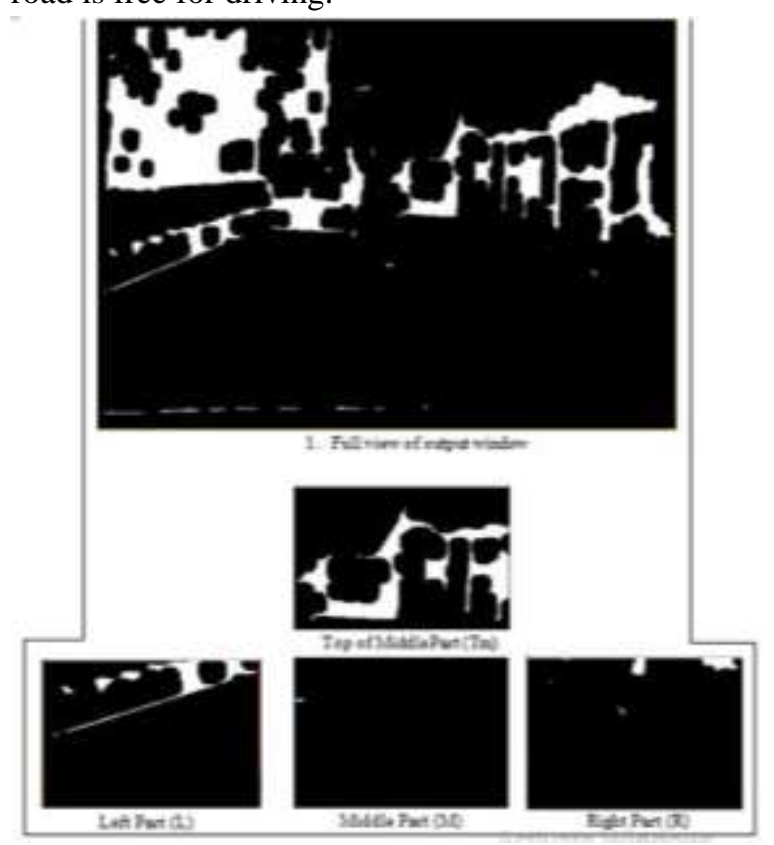

Fig.4. Car Tracking

If the white area is $2000>1<4000$ the object is detected but it is small, so alerts like "Watch Right or Left side" will be send to user's display. But if the white part is greater than 4500 or 5000 the system will first check in which part the intensity of white part are more and then alert the driver. If the intensity of traffic in both left and right is higher, then the "Please apply the brakes" alert will display. The speed of the vehicle can be suggested by calculating the white parts in middle and upper middle portion of video. As shown in fig.4, the top middle part (Tm) has more than 4500 white pixels that mean there is heavy vehicle in front of car but the middle part has no white portion so the driver will suggested driving at the speed of $30-40 \mathrm{~km} / \mathrm{hrs}$. If the intensity of white pixels is higher in both middle and upper middle part the driver will suggested to drive at the speed of 20-30 $\mathrm{km} / \mathrm{hrs}$. Using all this information in spite of heavy traffic the ADAS will alert the driver and keep car at safe distance.

\section{Road Detection}

Road detection is difficult in Indian cities because the size and structures of road changes from area to area. The poor lane marking and uneven surface on road also make the tracking difficult. The technologies which tracks lanes by the marking or by structure will be fail in this environment. The Proposed Advanced Driver Assistance System is tracking road and detecting drivable roads on the basis of edges and boundaries of road as shown in figure 5 . 


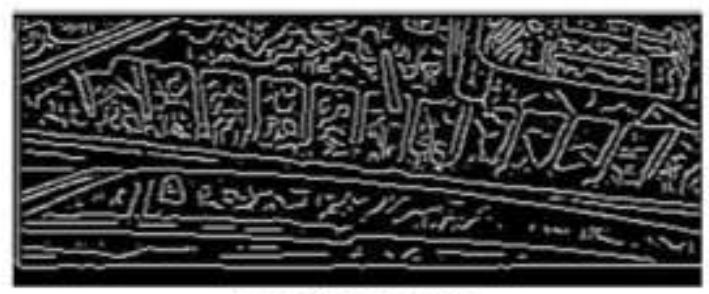

Fig.5 Edge detection

The colors cause the delusions and create error that's why the video is converted into gray scale. The Region of Interest is generated using U-Y selector. If any obstacle come in the RoI part of road the alert signal will send to driver. For more reliable output the $\mathrm{R}^{\prime} \mathrm{G}^{\prime} \mathrm{B}$ to $\mathrm{Y}^{\prime} \mathrm{Cb}$ ' $\mathrm{Cr}$ conversion done, as the human eyes are unable to see the chrominance. FIR filter is use to remove unwanted error and to stabilize the input video if the speed of vehicle is fast. Auto Threshold converts the gray scale/intensity image into binary image. For detection of line the Hough Transform is used. The Hough transform will calculate the theta and rho values from matrix and the connected points. The extended connecting points will detect as white solid line which indicate the boundary of road. The disconnected short line will detect as white broken line which indicate the edges of the driving lane as shown in figure 6 .

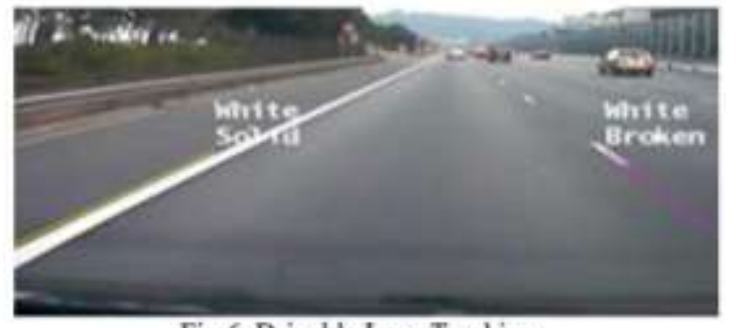

Fig.6. Drivable Lane Tracking

The Line Coordinator will calculate the distance between the lines in current frame and previous frame. The Kalman Filter is used for tracking this information. It utilizes the previous calculated state to project the current state. If car drift out of the driving lane or if other vehicle from any direction come near the vehicle, the system will give notification to the driver.

\section{Result}

The Experimental results are based on the output of Matlab Simulink showing the different driving scenario in Indian city. The figure 7 shows the different types of traffic conditions in Nagpur city in India. As the vehicle came in Region of Interest as shown in fig.7 (B) the system send warning to the driver. The intensity of the vehicle is large and it is too close to driving car. System first checked for alternative road for car but the road is small and forefront vehicle is large so "Break Applied" warning is given. Fig $7(\mathrm{C}),(\mathrm{F})$ shows the road edges and boundaries detection respectively. As the intensity of vehicle is small in $7(\mathrm{G})$, the alerts are given with respect to white part in the left part of video. This result clearly shows the detection of light as well as of heavy vehicle.

In figure 7 (D), system first checked the intensity of white parts in all parts. As the vehicle covered large part of the road there is very less place to overtake it. The edge detection gave the warning to watch left side of road as there is electrical poll and walkway which is close to car. If the car driver tried to overtake it would get crashed or accident could have happened which avoided using ADAS technology.

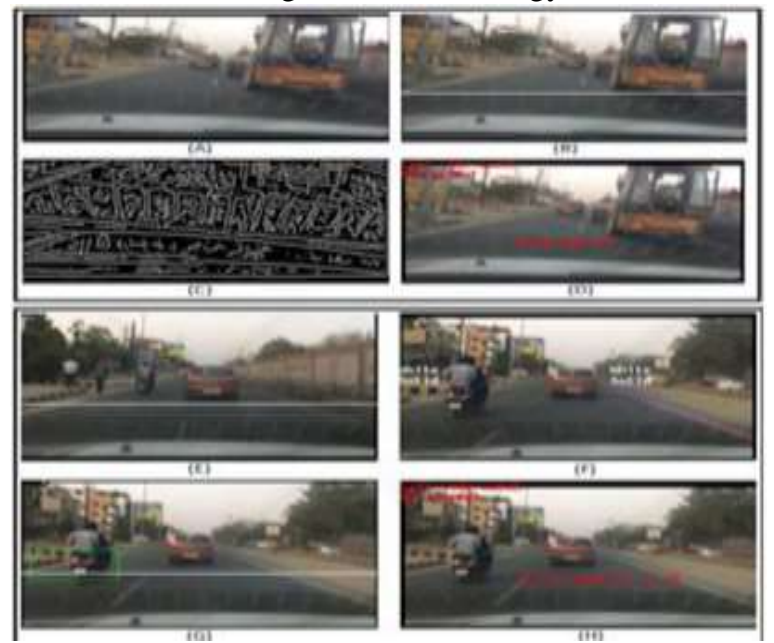

Fig.7 Experimental Result of Road Detection, Vehicle Detection and Alerts in City (A) Original Video Image (B) RoI Generation (C)Edge

Detection (D) Warning When Vehicle is too close (E) RoI Generation (F) Road Boundaries detection (G) Detection of Two Wheeler Warning and Alerts

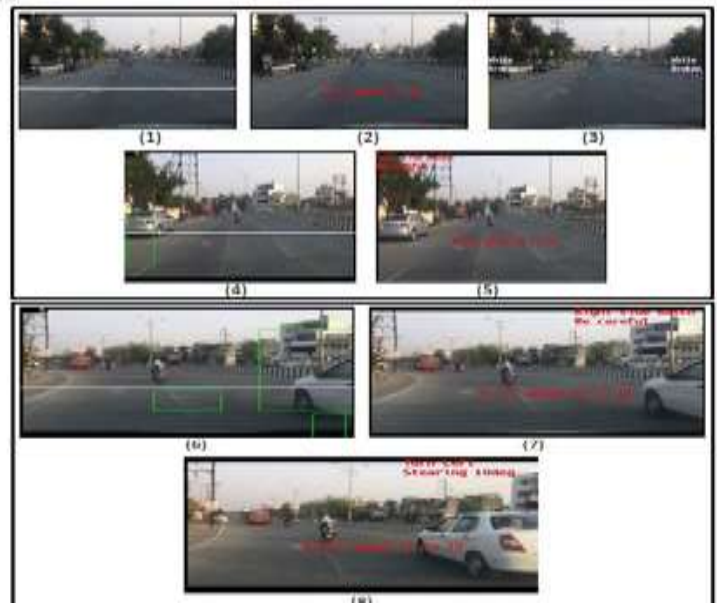

Fig. 8 Road and Side Vehicle Detection on Free Way (1) RoI Generated (2) Speed Suggestion (3) Road Boundaries Detection (4) Detection of Side Vehicle (5) Warning and Alerts (6) Detection of Side Vehicle (7) Warning (7) Alert 
Figure 8 shows the experimental results on free and spacious road. There is no detection of any obstacles in fig 8 (1) the speed limit is set to 45 to 60 kilometres per hour. The boundary of road is tracked using Hough transform and Kalman Filter as shown in figure 8 (3). The intensity of white part is increased in 8 (4) because there is side vehicle on the road hence the alerts are send to driver as shown in figure 8 (5). Fig.8 (6) shows the successful detection of side vehicle at the turning of the road. The direction and angle of other vehicle calculated on basis of the white part intensity. The graph shown in figure 9 shows the comparison of the traffic intensity in early morning when there is less congestion and of office time when roads are full congested.

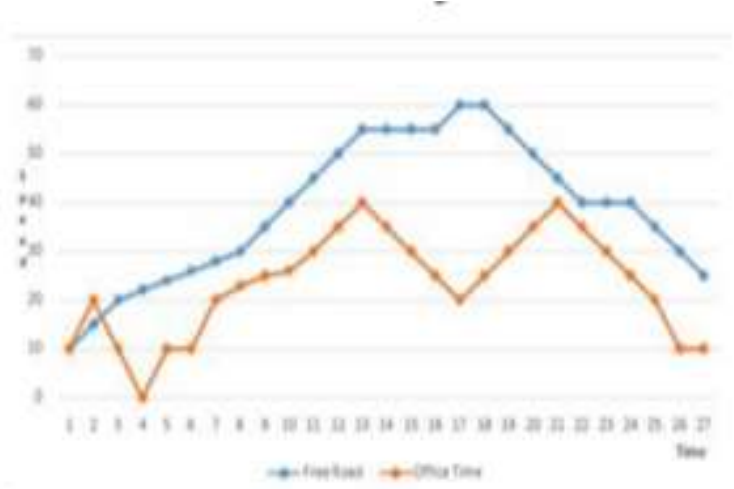

Fig 9: Comparison of Traffic Intensity of Early Morning and Office Time

\section{Conclusion}

In this paper, the presentation of Advanced Driver Assistance System especially for Indian Road scenario is shown. The result shows the systematic tracking and detection of roads, vehicle in all scenarios by boosting the capabilities like ACC (Adaptive Cruise Control), LKA (Lane Keep Assist), and PCS (Pre-crash System). In spite of structure difficulties, chaotic traffic and congested roads the system worked properly and alert driver whenever objects detected in region of interest.

The system can be loaded with other function like Automatic emergency breaking, Tire pressure warning system, Parking cameras and sensors, Temperature sensor, Heat sensor, Smoke Detector etc. For more accurate results the sensors like Millimeter wave radar Sensor (MMW), Engine Coolant temp (ECT), Wheel Speed Sensor, Tire Pressure sensor (TPMS) etc. can be implemented on the vehicle which will give the accurate on-time calculations.

\section{References}

[1] http://sites.ndtv.com/roadsafety/importantfeature-to-you-in-your-car-5/

[2] https://www.google.co.in/
[3] http://meity.gov.in/content/intelligenttransportation-system-its

[4] Rijurekha Sen, Bhaskaran Raman, "Intelligent Transport Systems for Indian Cities", Department of Computer Science and Engineering Indian Institute of Technology, Bombay, 2011

[5] Advanced Driver Assistance System, RavindraB.S and Vijaykumar K.V, Mistral Solutions ptv, lmt. India

[6] Chunzhao Guo, Junichi Meguro, Yoshiko Kojima, and Takashi Naito, "CADAS: a Multimodal Advanced Driver Assistance System for Normal Urban Streets based on Road Context Understanding,'IEEE Intelligent Vehicles Symposium (IV), 2Gold Coast, Australia June 23-26, 2013

[7] Paul George, Indira Thouvenin, Vincent Fr'emont and V'eronique Cherfaoui, "DAARIA: Driver Assistance by Augmented Reality for Intelligent Automobile", Intelligent Vehicles Symposium Alcalá de Henares, Spain, June 3-7, 2012

[8] Yen-Lin Chen, Hsin-Han Chiang, Chuan-Yen Chiang, Chuan-Ming Liu, Shyan-Ming Yuan and Jenq-Haur Wang, “A Vision-Based Driver Nighttime Assistance and Surveillance System Based on Intelligent Image Sensing Techniques and a Heterogamous Dual-Core Embedded SystemArchitecture",,www.mdpi.com/journal/s ensors, Sensors 2012

[9] Zehang Sun, George Bebis2 and Ronald Miller3, On-Road Vehicle Detection Using Optical Sensors: A Review, 1eTreppid Technologies, LLC, Reno, NV2Computer Vision Laboratory, University of Nevada, Reno, NV 3Vehicle Design R \& A Department, Ford Motor Company, Dearborn, MI.

[10] Andreas Festag, Alban Hessler, Roberto Baldessari, Long Le, Wenhui Zhang, Dirk Westhoff, "VEHICLE-TO-VEHICLE AND ROAD-SIDE SENSOR COMMUNICATION FOR ENHANCED ROAD SAFETY", NEC Laboratories Europe, Network Research DivisionKurf ursten-Anlage 36, D-69115 Heidelberg

[11]Christos Katrakazas, Mohammed Quddus, Wen-Hua Chen, Lipika Deka, "Real-time motion planning methods for autonomous onroad driving: State-of-the-art and future research directions", Transportation Research Part C 60 (2015) 416-442 